Delft University of Technology

\title{
Impact of Crude Oil on Pre-Generated Foam in Porous Media
}

Hussain, A. A.A.; Vincent-Bonnieu, S.; Kamarul Bahrim, R. Z.; Pilus, R. M.; Rossen, W. R.

DOI

10.1016/j.petrol.2019.106628

Publication date

2020

Document Version

Final published version

Published in

Journal of Petroleum Science and Engineering

\section{Citation (APA)}

Hussain, A. A. A., Vincent-Bonnieu, S., Kamarul Bahrim, R. Z., Pilus, R. M., \& Rossen, W. R. (2020). Impact of Crude Oil on Pre-Generated Foam in Porous Media. Journal of Petroleum Science and Engineering, 185 , [106628]. https://doi.org/10.1016/j.petrol.2019.106628

\section{Important note}

To cite this publication, please use the final published version (if applicable).

Please check the document version above.

\section{Copyright}

Other than for strictly personal use, it is not permitted to download, forward or distribute the text or part of it, without the consent of the author(s) and/or copyright holder(s), unless the work is under an open content license such as Creative Commons.

\section{Takedown policy}

Please contact us and provide details if you believe this document breaches copyrights.

We will remove access to the work immediately and investigate your claim. 


\title{
Impact of Crude Oil on Pre-Generated Foam in Porous Media
}

\author{
A.A.A. Hussain ${ }^{\text {a, }}$, S. Vincent-Bonnieu ${ }^{\text {a,b }}$, R.Z. Kamarul Bahrim ${ }^{c}$, R.M. Pilus ${ }^{\text {d }}$, W.R. Rossen ${ }^{\text {a }}$ \\ ${ }^{\text {a }}$ Department of Geoscience and Engineering, Delft University of Technology, Delft, Netherlands \\ ${ }^{\mathrm{b}}$ Shell Global Solutions International B.V., Amsterdam, Netherlands \\ ${ }^{\mathrm{c}}$ PETRONAS Research Sdn Bhd, Bandar Baru Bangi, Malaysia \\ ${ }^{\mathrm{d}}$ Department of Petroleum Engineering, Universiti Teknologi PETRONAS, 32610, Bandar Seri Iskandar, Perak, Malaysia
}

\section{A R T I C L E I N F O}

\section{Keywords:}

Foam

Porous medium

Crude oil

Surfactant

Enhanced oil recovery

EOR

\begin{abstract}
A B S T R A C T
As foam is injected into an oil reservoir, the region near an injector can become oil-free due to the relatively high capillary number. Foam created in this region encounters oil further out in the reservoir. The impact of oil on foam in porous media is usually investigated by co-injecting surfactant, gas and oil, or by injecting pre-generated foam into an oil-saturated core. However, the former experiment does not give information on the impact of oil on pre-generated foam, and with the latter experiment one cannot easily obtain data at different oil fractional flows, necessary to model the impact of oil on pre-generated foam.

Here we present a novel but relatively simple experimental technique for investigating the effect of oil on pregenerated foam in porous media. This allows one to compare the effects of oil on pre-generated foam and on foam generation without the experimental complications involved in using two separate porous media. We coinject surfactant solution and gas into a relative narrow core $(1 \mathrm{~cm}$ diameter), and inject oil into the core some distance downstream from the inlet through ports in the side of the core. The relatively narrow core allows rapid contact between the injected crude oil and pre-generated foam in a porous medium much larger than pore dimensions. By injecting the three phases into the core we investigate the flow behaviour of foam with oil at fixed fractional flows of all three phases.

We illustrate the technique with a study of the effect of one crude oil with two surfactants. With this system, there is a progressive decrease in the apparent viscosity of the foam after encountering oil. Foams with a higher gas fraction experiences a more-significant weakening by oil over the length of the core than foams with a lower gas fraction. By the end of the core, the apparent viscosities of foam with a higher gas fraction approach values observed with three-phase co-injection. We present a novel, but relatively simple method to investigate the change of foam mobility as it encounters oil in a porous medium, at controlled fractional flows. We show that in our case the apparent viscosity of foam with oil can decrease by more than a factor of four over a distance of $15 \mathrm{~cm}$, indicating that foam and oil reach steady-state (as observed with three-phase co-injection) almost instantaneously compared to the length of a reservoir-simulation grid-block.
\end{abstract}

\section{Introduction}

Most research on the impact of (crude) oil on foam in porous media is conducted applying one of the following six methods:

Co-injection of oil, gas, and surfactant solution into a core (Hussain et al., 2019; Tang et al., 2018).

Injection of pre-generated foam injection into a core pre-saturated with oil (Aarra and Skauge, 1994; Kristiansen and Holt, 1992; Tang, 2019).
Co-injection of gas and surfactant solution into a core (partly) presaturated with oil (Raza, 1970; Simjoo, 2012).

Co-injection of gas and surfactant solution into a core which is at waterflood-residual oil saturation (Janssen et al., 2019a, 2019b). Injection of surfactant solution followed by gas into a core which is at waterflood-residual oil saturation (Nikolov et al., 1986).

Injection of pre-generated foam into a microfluidics chip, where oil is injected some distance downstream from the main inlet (Schramm et al., 1993; Schramm and Novosad, 1990).

\footnotetext{
* Corresponding author.

E-mail address: ahmed_hussain@outlook.com (A.A.A. Hussain).
} 
Table 1

Synthetic seawater composition.

\begin{tabular}{ll}
\hline Salts & Grams/litre \\
\hline $\mathrm{NaCl}$ & 25.4 \\
$\mathrm{KCl}$ & 0.673 \\
$\mathrm{MgCl}_{2} \cdot 6 \mathrm{H} 2 \mathrm{O}$ & 10.2 \\
$\mathrm{CaCl}_{2} \cdot 2 \mathrm{H} 20$ & 1.47 \\
$\mathrm{Na}_{2} \mathrm{SO}_{4}$ & 3.83 \\
\hline
\end{tabular}

However, these processes do not necessarily represent what happens with the application of foam for enhanced oil recovery (EOR), where foam can sometimes be generated in the absence of oil near the well. This (pre-)generated foam then propagates into regions richer in oil, where the different phases interact.

A difference in flow characteristics between co-injection of three separate phases and co-injection pre-generated foam and oil arises from the difference in how oil impacts foam, i.e. by anti-foaming and/or defoaming. Anti-foamers inhibit foam formation, and de-foamers destabilize an existing foam. For bulk foams outside porous media, defoamers usually act on the outer surface of the foam (Pugh, 1996). By co-injecting gas, surfactant solution and oil, it is possible that foam is not created, due to strong anti-foaming impact by the oil. Tang (2019) reports completely different behaviour when injecting pre-generated foam than when co-injecting surfactant solution and gas into a core at waterflood-residual oil saturation. This indicates that, as with bulk foam, the impact of oil on pre-generated foam can be different from its impact on foam generation. By injecting foam pre-generated outside the porous medium, there is an uncertainty whether the characteristics of the injected foam are the same as for in-situ-generated foam, especially if the foam generator has different properties than the core (Falls et al., 1989). This approach is also complicated by the capillary end effect at the outlet face of the first porous medium and coarsening of foam during transport to the second. Co-injecting both oil and pre-generated foam at the core inlet can result in oil weakening the foam at the T-junction of the apparatus tubing or in the injection plate.

Therefore, we choose to investigate the impact of crude oil on foam by co-injecting surfactant solution and gas from the face of the core, and oil some distance downstream from the coreface, to investigate the impact of crude oil on in-situ pre-generated foam. This is similar in intent to the experiments conducted by Schramm and Novosad (1990) in glass micromodels. For foams that are weakened by oil, they report that the foam lamellae transported oil droplets for some distance before rupturing, after which the following lamellae picked up and transported the oil droplets. For an overview of the impact of oil on foam in porous media see Farajzadeh et al. (2012).

In the next section of this paper we present an overview of the materials we use for our experiments and experimental procedures. Using a relatively narrow core allows rapid contact between injected oil and foam, in a realistic porous medium much larger than pore dimensions. That section is followed by an overview and discussion of our experimental findings and then our conclusions.

\section{Materials and procedure}

We use anionic surfactant C14/16 Alpha Olefin Sulfonate (AOS, brand-name Witconate, supplied by AkzoNobel) and a proprietary mixture of anionic and amphoteric surfactants, referred to as surfactant A. We prepare all the surfactant solutions with $0.5 \mathrm{wt} \%$ surfactant concentration. The surfactant solution is based on a synthetic seawater solution; see Table 1 for its composition. For AOS the critical micelle concentration (CMC) is roughly $0.003 \mathrm{wt} \%$ at $23 \mathrm{C}$ (Jones et al., 2016a). To satisfy adsorption, the core is flooded with more than 10 pore volumes of surfactant solution before conducting experiments. Nitrogen gas is injected into the core with a purity of $99.98 \%$, supplied from a 200-bar gas cylinder. The crude oil has a viscosity of $3.8 \quad 0.03 \mathrm{cP}$ and

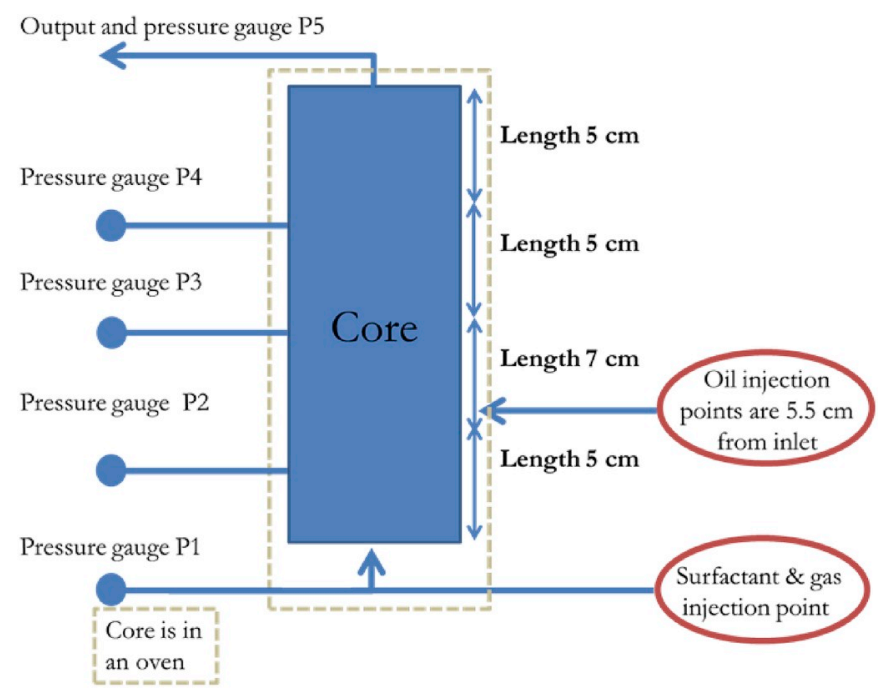

Fig. 1. Schematic of the apparatus used for this experiment. Note that there are two oil-injection points $5.5 \mathrm{~cm}$ from the main inlet on opposite sides of the core. (Only one is shown here to avoid clutter.)

density of $0.84 \quad 0.01 \mathrm{~g} / \mathrm{cm}^{3}$, measured at $20 \mathrm{C}$.

For our experiments with AOS we use Bentheimer sandstone, which has a porosity of about 0.25 (Peksa et al., 2015). By water-flooding the core we determined the permeability, $\mathrm{k}$, to be $2.6 \quad 0.2 \quad 10^{12} \mathrm{~m}^{2}$ (2.6 0.2 D). (The range represents the standard deviation of the fit.) The experiments with surfactant A are conducted with Berea sandstone, which has a porosity of about 0.2 (Kapetas et al., 2015; Øren and Bakke, 2003). By pumping water through a water-saturated core we determined the permeability to be $0.13 \quad 0.005 \quad 10^{12} \mathrm{~m}^{2}\left(\begin{array}{lll}130 & 5 \mathrm{mD}\end{array}\right)$. The cores are $22 \mathrm{~cm}$ in length and are $1 \mathrm{~cm}$ in diameter. The cores are coated in epoxy resin, which results in an effective core diameter of $0.94 \mathrm{~cm}$, and are mounted in aluminium core-holders, as was done by Jones et al. (2016a, 2016b).

Nitrogen and surfactant solution are injected from the bottom coreface, reached through relatively narrow tubes and connections, with an inner diameter of $0.75 \mathrm{~mm}$, to minimize the droplet size of the entering phases. Oil is injected $5.5 \mathrm{~cm}$ from the main inlet; see Fig. 1. We measure the pressure along the length of the core with five pressure gauges, located $0,5,12,17$ and $22 \mathrm{~cm}$ from the main inlet. In this text we refer to the portion of the core between two adjacent pressure gauges as

Table 2

The measured and calculated surfactant and oil content of the surfactant solutions used.

\begin{tabular}{|c|c|c|c|c|}
\hline Description & $\begin{array}{l}\text { Total c } \\
(\mathrm{ppm})\end{array}$ & arbon & $\begin{array}{l}\text { Oil content } \\
\text { (wt.\%) }\end{array}$ & $\begin{array}{l}\text { Surfactant concentration } \\
\text { (wt.\%) }\end{array}$ \\
\hline $\begin{array}{l}\text { Initial AOS } \\
\text { solution }\end{array}$ & 2878 & $140^{\mathrm{a}, \mathrm{b}}$ & - & $0.50 \quad 0.02^{\mathrm{a}, \mathrm{b}}$ \\
\hline $\begin{array}{l}\text { AOS solution } \\
\text { with } \\
\text { solubilized } \\
\text { crude oil }\end{array}$ & 3329 & $140^{\mathrm{b}}$ & $0.14 \quad 0.02^{b, c}$ & $\begin{array}{l}0.370 .01^{c} \text {; difference } \\
\text { assumed due to surfactant } \\
\text { lost to oil-water emulsion }\end{array}$ \\
\hline
\end{tabular}

${ }^{\text {a }}$ Values calculated with the active content in the initial AOS solution.

b Values calculated with Shimadzu TOC analyser values.

c Values calculated with the surfactant titration measurement.

Table 3

Surface-tension values measured at ambient conditions.

\begin{tabular}{lll}
\hline Surface tension $(\mathrm{mN} / \mathrm{m})$ & & \\
\hline Crude oil & 27 & 1 \\
Synthetic seawater & 73 & 1 \\
Synthetic seawater with 0.5 wt $\%$ AOS C14-16 & 28 & 1 \\
\hline
\end{tabular}


Table 4

Interfacial-tension values measured at ambient conditions, and the calculated entering, spreading and bridging coefficients, and lamella number. The measured interfacial tension between crude oil and surfactant solution was below the measurement range of the device $(1-350 \mathrm{mN} / \mathrm{m})$. For our calculations of the range of possible values of the foam-stability coefficients in Table 3 we use an interfacial tension of 0 and $1 \mathrm{mN} / \mathrm{m}$.

\begin{tabular}{clllll}
\hline & $\begin{array}{l}\text { Interfacial } \\
\text { tension } \\
(\mathrm{mN} / \mathrm{m})\end{array}$ & $\begin{array}{l}\text { Entering } \\
\text { coefficient }\end{array}$ & $\begin{array}{l}\text { Spreading } \\
\text { coefficient }\end{array}$ & $\begin{array}{l}\text { Bridging } \\
\text { coefficient }\end{array}$ & $\begin{array}{l}\text { Lamella } \\
\text { number }\end{array}$ \\
\hline $\begin{array}{c}\text { Crude oil/ } \\
\text { synthetic }\end{array}$ & $<1$ & $28-29$ & $1-2$ & $95-96$ & $4-\infty$ \\
seawater & & & & & \\
$0.5 \mathrm{wt}$ & & & & & \\
$\%$ AOS & & & & \\
C14-16 & & & & \\
\hline
\end{tabular}

sections. The main inlet is located in section 1 , section 2 is the following section, and so forth. For the experiments with AOS at 50, 70 and $95 \%$ foam quality, the oil was injected with a single syringe pump from a single injection port; for all the other experiments with two syringe pumps from two different injection ports. The relatively narrow core allows rapid contact between the injected crude oil and pre-generated foam, especially when oil is injected from both sides. The experiments were conducted at a controlled temperature of $30 \mathrm{C}$ with AOS, and at $90 \mathrm{C}$ with surfactant $\mathrm{A}$, and both with a back-pressure of 40 bar.

Table 3 gives the surface tensions of crude oil, synthetic seawater and the surfactant solution. Interfacial-tension (IFT) values of $<1 \mathrm{mN} / \mathrm{m}$ and $181 \mathrm{mN} / \mathrm{m}$ were measured between crude oil and surfactant solution and synthetic seawater, respectively. These measurements were conducted using the Du Noüy-Padday method at room temperature (21 $1 \mathrm{C}$ ) and ambient pressure. Table 4 gives the relevant interfacial tensions for the crude oil and the aqueous solutions, and the respective values of entering, spreading, and bridging coefficients and lamella number. These values were calculated as described in Farajzadeh et al. (2012). The positive value for the entering coefficient indicates that AOS foam is unstable in presence of the crude oil.

We prepare our surfactant solution with solubilized oil as follows: we mix AOS surfactant solution (1029.1 $0.1 \mathrm{~g}$ ) and crude oil (198.9 $0.1 \mathrm{~g}$ ) and stir daily for 11 days. We then separate the surfactant solution from the crude oil and emulsion layer by using a separation funnel. To remove

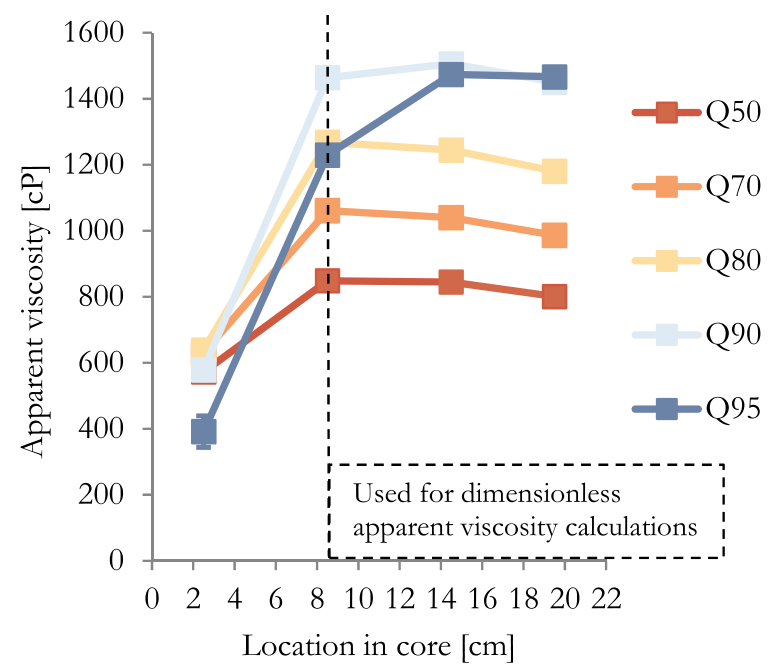

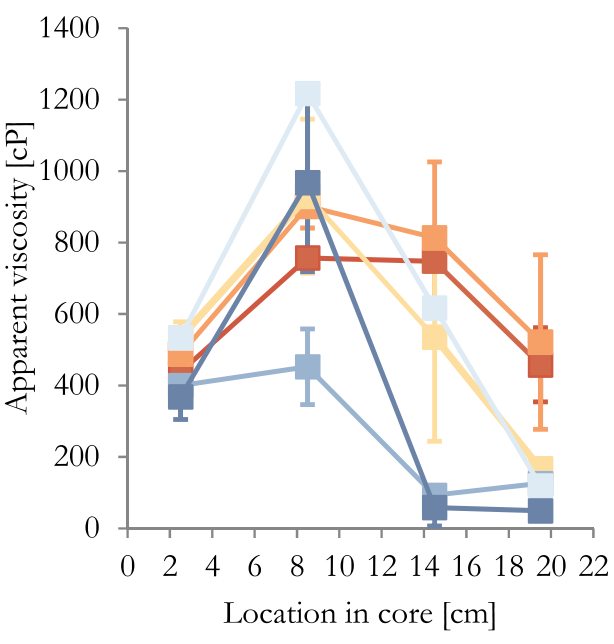

$\mathrm{B}$

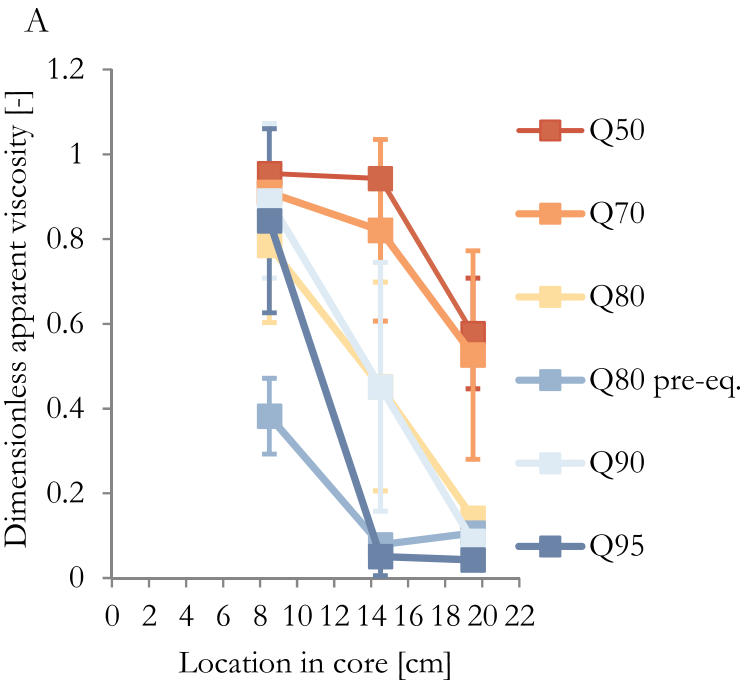

C

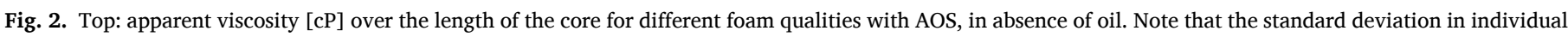

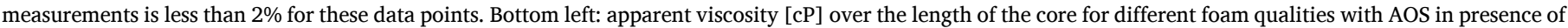

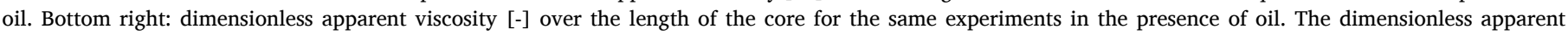

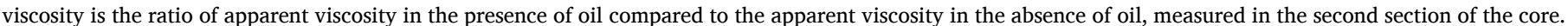

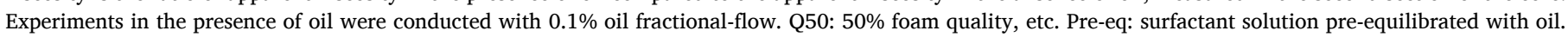




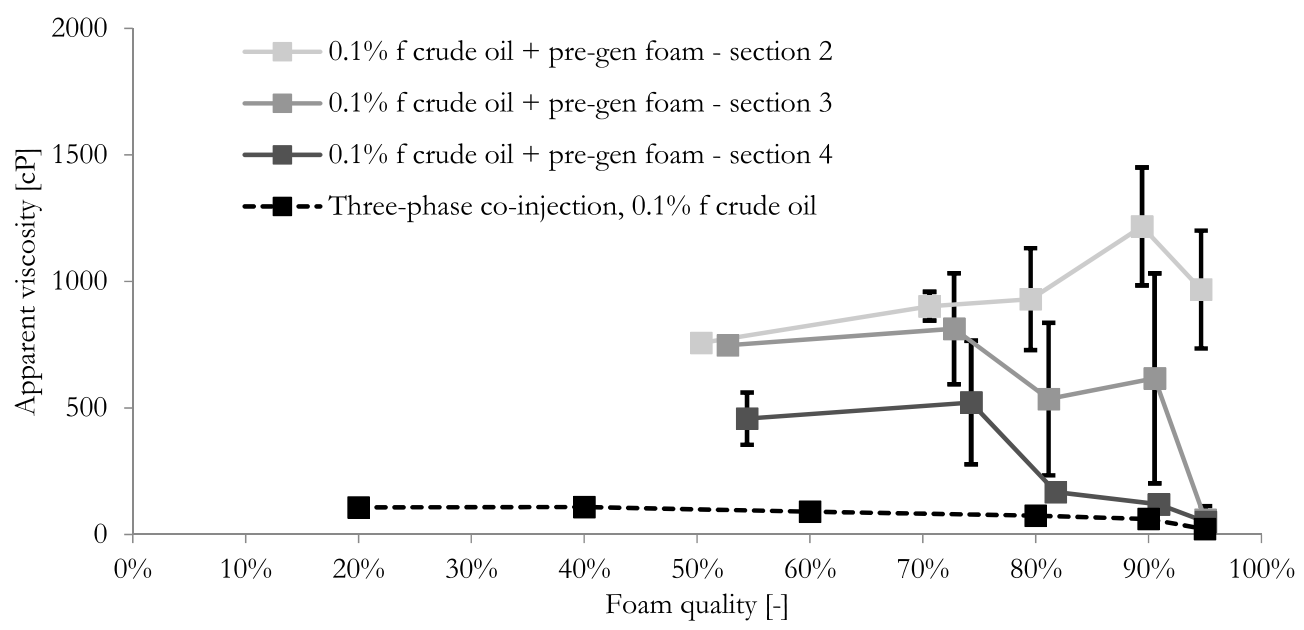

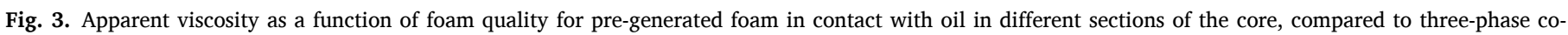

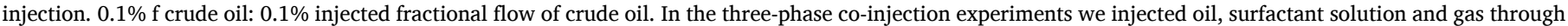
the same injection port. These experiments were conducted with the same materials as other experiments, except with a shorter core (17 $\mathrm{cm}$ vs. $22 \mathrm{~cm})$.

any emulsion droplets from the surfactant solution, we centrifuge the solution at $2000 \mathrm{rpm}$ for $2 \mathrm{~h}$, and finally filter through a filter paper (Sartorius) with a pore size of $0.45 \mu \mathrm{m}$, under a pressure gradient imposed by a vacuum pump. The surfactant concentration in the surfactant solution equilibrated with crude oil was determined by titration to be $0.370 .01 \mathrm{wt} \%$. From Total Oil Content (TOC) measurements (using a Shimadzu TOC analyser and a Skalar PrimacsSLC TOC analyser), we deduce that the solubilized crude oil content was $0.140 .03 \mathrm{wt}$ $\%$; see Table 2 . We assume that the reduction in surfactant concentration by $0.13 \mathrm{wt} \%$ detected by titration reflects surfactant consumption by emulsions or solubilisation into the oil when equilibrating the surfactant solution.

Core-flood experiments with both AOS and surfactant A were conducted with a total injection rate of $0.1 \mathrm{ml} / \mathrm{min}$ and $0.02 \mathrm{ml} / \mathrm{min}$ respectively, which is equivalent to superficial velocities of $6.8 \mathrm{ft} / \mathrm{day}$ and $2 \mathrm{ft}$ /day. To minimize any impact of hysteresis while conducting the foam-quality scan, in collecting data we alternate between lower and higher foam qualities. We define foam quality as the gas fraction of the combined gas and water injection rate (i.e., excluding oil).

After we reach steady-state in an experiment, we start to prepare the core for the following experiment. To achieve an oil saturation greater than what will be achieved with the subsequent experiment we stop gas injection but continue injection of surfactant solution at $0.001 \mathrm{ml} / \mathrm{min}$ to prevent oil moving upstream. We inject at least $3 \mathrm{ml}$ of oil (at $0.05 \mathrm{ml}$ / $\min$ ), more than one pore volume of the three downstream sections. After injecting $3 \mathrm{ml}$ of oil we inject foam and oil at the desired foam quality and oil fractional flow. This experimental procedure allows us to investigate the steady-state behaviour of pre-generated foam in the presence of crude oil at various oil fractional flows and initial oil saturations.

\section{Results and discussions}

In each section of the core we calculate the "apparent viscosity" from the average pressure gradient over that section and assuming singlephase flow; see eq. (1). Here $\mu_{\text {app }}$ is the apparent viscosity [cP], $\mathrm{k}$ is permeability $\left[\mathrm{m}^{2}\right], \mathrm{u}$ is superficial velocity $[\mathrm{m} / \mathrm{s}]$, and $\nabla \mathrm{P}$ is the pressure gradient over that section. We define the dimensionless apparent viscosity as the ratio of apparent viscosities observed with pre-generated foam in the presence of oil to the apparent viscosity observed in section 2 in the absence of oil.

$\mu_{a p p} \quad \frac{k}{u} \nabla P$

\subsection{AOS foam and crude oil}

Fig. 2A and B show the dimensional and dimensionless apparent viscosities, respectively, as a function of position along the core, for

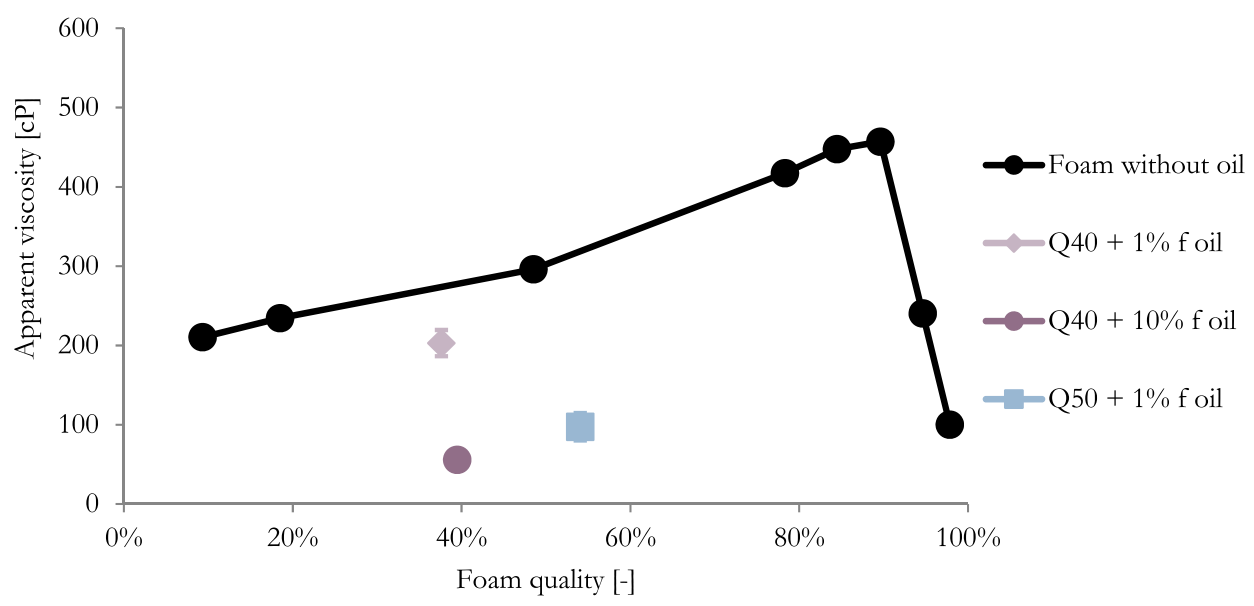

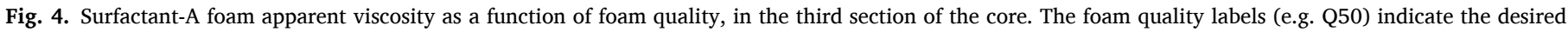

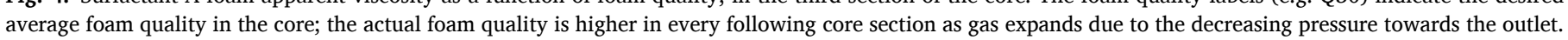




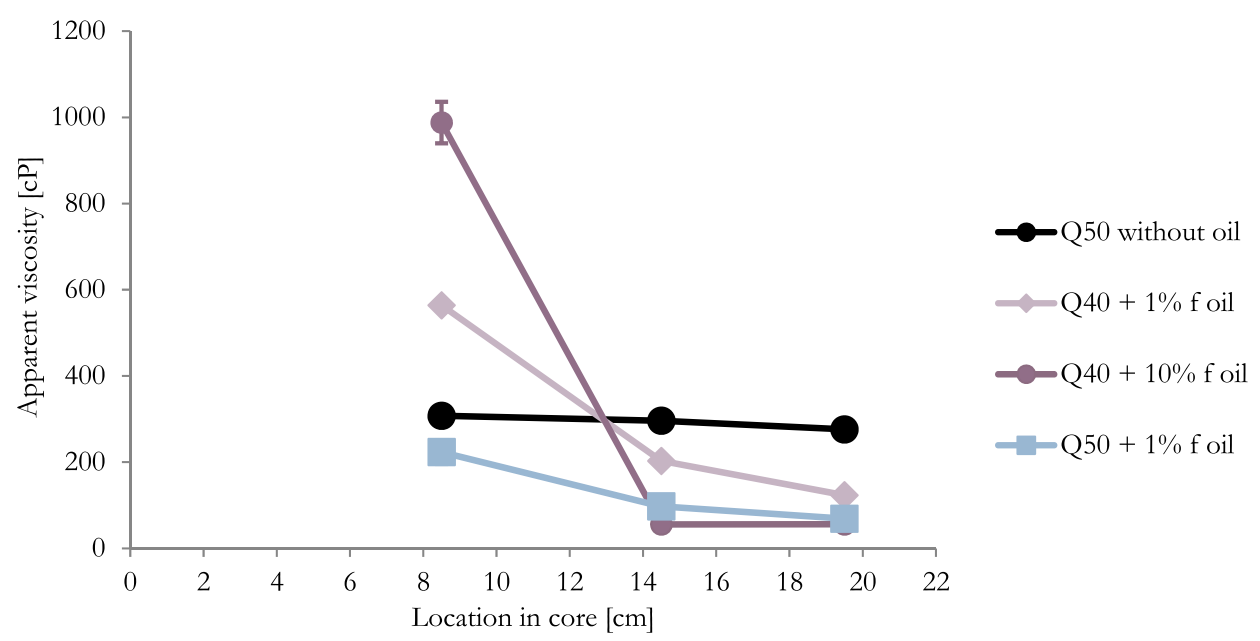

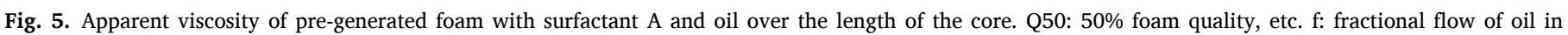
injected fluids.

different foam qualities with AOS surfactant. AOS foam progressively weakens after it comes into contact with crude oil. Higher-quality foams experience a steeper and greater decline in apparent viscosity over the length of the core than lower-quality foams. In the second section of the core, the apparent viscosity of $80 \%$ quality foam, generated with preequilibrated surfactant $(453 \mathrm{cP})$, is significantly lower compared to that of foam generated with surfactant which hadn't previously been in contact with oil $(930 \mathrm{cP})$. However, apparent viscosity at the end of the core is more similar for foam with pre-equilibrated surfactant $(126 \mathrm{cP})$ and with surfactant which hadn't previously been in contact with oil $(167 \mathrm{cP})$.

Fig. 3 shows apparent viscosity as a function of foam quality in the different sections of the core. It also shows the apparent viscosity observed in three-phase co-injection experiments, where the oil, surfactant solution and gas are injected from the same port, with a total velocity superficial velocity of $6.8 \mathrm{ft} / \mathrm{day}$. Compared to the other reported experiments here, the three-phase co-injection experiments were conducted with the same materials and set-up, except with a shorter core $(17 \mathrm{~cm}$ vs. $22 \mathrm{~cm})$. The apparent viscosities in that case are calculated over a section starting $5.25 \mathrm{~cm}$ from the inlet to $5.25 \mathrm{~cm}$ from outlet of the core. As foam propagates through the core, the apparent viscosity gradually decreases. We believe that, in a sufficiently long core, apparent viscosities with pre-generated foam and oil would approach those with three-phase co-injection. It is unclear why apparent viscosity increases in the last section of the core with pre-equilibrated foam (Fig. 2), as we did not observe this in any other experiments.

These results are consistent with the results of Schramm and Novosad (1990), who showed that foam lamellae in micromodels can travel some distance with oil droplets in them before rupturing. This indicates that pre-generated foam that comes into contact with oil in a porous medium does not necessarily collapse instantaneously, and can travel some distance on the core scale. Aarra and Skauge (1994) and Kristiansen and Holt (1992) conducted similar experiments to these, where they pre-generated AOS-foam with qualities from $65 \%$ to $95 \%$ outside their core and injected the foam into a core with crude oil at a residual saturation. As we do, they observed decreasing apparent viscosity along the length of the core for foam qualities between $65 \%$ and $95 \%$ in the presence of oil. Their results, together with ours, show that the impact of crude oil on pre-generated foam is a function not only of oil saturation and fractional flow, but also a function of foam quality.

\subsection{Surfactant $A$ foam and crude oil}

Figs. 4 and 5 show the apparent viscosity of foam with surfactant A in the absence of oil and of pre-generated foam in the presence of oil, respectively, over the length of the core. Similar to the experiments with AOS, the apparent viscosity decreases progressively after the first contact of the pre-generated foam with the crude oil. However, unlike the experiments with AOS, an abrupt increase in apparent viscosity is observed as the pre-generated foam first contacts oil. Figs. 4 and 5 show that the apparent viscosity observed at first contact of oil and 40\%quality foam increases with an increasing fractional-flow of oil from 0 to $10 \%$. We believe that the increase in apparent viscosity at first contact with oil reflects the combined effects of reduced relative permeabilities in three-phase flow and emulsion generation. Emulsion was observed in the core effluent in this experiment. After first contact, a steeper decrease in apparent viscosity occurs with $10 \%$ oil fractional flow than with $1 \%$. This is consistent with observations in microfluidics by Schramm and Novosad (1990), who showed that foam is weakened by oil droplets carried in the foam lamellae. Greater oil fractional flow means there is more oil in contact with the foam to destabilize it.

\section{Conclusions}

We present a novel experimental approach to investigate the impact of oil on pre-generated foam at controlled oil flow rate. This approach allows one to investigate the weakening of pre-generated foam by oil as a function of distance travelled. Separate oil injection allows generation of foam without oil in the same porous medium before first contact with oil. The relatively narrow core diameter ensures rapid contact between foam and injected oil.

For the crude oil and surfactants examined here, pre-generated foam progressively weakened in presence of crude oil after first contact. The apparent viscosity in some cases decreased by more than a factor four over a distance of $15 \mathrm{~cm}$. We believe that in a sufficiently long core the pre-generated foam in contact with oil would gradually weaken until reaching the same apparent viscosity as with three-phase co-injection. With surfactant A there is an increase in apparent viscosity as foam first encounters the crude oil, before progressively weakening. We believe this reflects reduced gas and water relative permeabilities in three-phase flow, and possibly emulsification of oil in water.

After coming into contact with oil, lower-quality foams progressively weakened over a somewhat longer distance, compared to higher-quality foams, until they reached their steady state apparent viscosity. This indicates that lower-quality foams are less susceptible (or less rapidly susceptible) to weakening by crude oil. (Distances in all cases are of course very short on a field scale.) In our experiments with $80 \%$ foam quality, we observe that foam made with surfactant pre-equilibrated with the crude oil reach their steady state apparent viscosity over a shorter distance in presence of oil compared to foam made with 
surfactant that hasn't contacted oil before.

\section{Acknowledgements}

The authors thank and acknowledge both the sponsorship and advice of Universiti Teknologi Petronas. Ridhwan Kamarul Bahrim would like to thank PETRONAS for their permission for publication. Sebastien Vincent-Bonnieu would like to thank Shell Global Solutions International B.V. especially for their permission for publication. In addition, we thank Michiel Slob, Merit van der Lee, Ellen Meijvogel-de Koning and Jolanda van Haagen-Donker for their expert technical assistance with our experiments.

\section{Nomenclature}

$\mathrm{K} \quad$ permeability $\left[\mathrm{m}^{2}\right]$

$\mathrm{U} \quad$ superficial velocity $[\mathrm{m} / \mathrm{s}]$

$\nabla \mathrm{P} \quad$ pressure gradient over section $[\mathrm{Pa} / \mathrm{m}]$

$\mu_{\text {app }} \quad$ the apparent viscosity [cP]

\section{Appendix A. Supplementary data}

Supplementary data to this article can be found online at https://doi. org/10.1016/j.petrol.2019.106628.

\section{References}

Aarra, M.G., Skauge, A., 1994. A foam pilot in a North Sea oil reservoir: preparation for a production well treatment. In: Presented at the SPE Annual Technical Conference and Exhibition. Society of Petroleum Engineers.

Falls, A.H., Musters, J.J., Ratulowski, J., 1989. The apparent viscosity of foams in homogeneous bead packs. SPE Reserv. Eng. 4, 155-164. https://doi.org/10.2118/ 16048-PA.

Farajzadeh, R., Andrianov, A., Krastev, R., Hirasaki, G.J., Rossen, W.R., 2012. Foam-oil interaction in porous media: implications for foam assisted enhanced oil recovery. Adv. Colloid Interface Sci. 183-184, 1-13. https://doi.org/10.1016/j. cis.2012.07.002.

Hussain, A.A.A., Vincent-Bonnieu, S., Kamarul Bahrim, R.Z., Pilus, R.M., Rossen, W.R., 2019. The impacts of solubilized and dispersed crude oil on foam in a porous medium. Colloids Surf. Physicochem. Eng. Asp. 579, 123671. https://doi.org/ 10.1016/j.colsurfa.2019.123671.
Janssen, M.T.G., Pilus, R.M., Zitha, P.L.J., 2019. A comparative study of gas flooding and foam-assisted chemical flooding in bentheimer sandstones. Transp. Porous Media. https://doi.org/10.1007/s11242-018-01225-3.

Janssen, M.T.G., Zitha, P.L.J., Pilus, R.M., 2019. Oil recovery by alkaline/surfactant/ foam flooding: effect of drive-foam quality on oil-bank propagation. SPE J. https:// doi.org/10.2118/190235-PA.

Jones, S.A., Laskaris, G., Vincent-Bonnieu, S., Farajzadeh, R., Rossen, W.R., 2016. Effect of surfactant concentration on foam: from coreflood experiments to implicit-texture foam-model parameters. J. Ind. Eng. Chem. 37, 268-276. https://doi.org/10.1016/j. jiec.2016.03.041.

Jones, S.A., van der Bent, V., Farajzadeh, R., Rossen, W.R., Vincent-Bonnieu, S., 2016 Surfactant screening for foam EOR: correlation between bulk and core-flood experiments. Colloids Surf. Physicochem. Eng. Asp. 500, 166-176. https://doi.org/ 10.1016/j.colsurfa.2016.03.072.

Kapetas, L., Vincent-Bonnieu, S., Farajzadeh, R., Eftekhari, A.A., Mohd-Shafian, S.R., Bahrim, R.Z.K., Rossen, W.R., 2015. Effect of permeability on foam-model parameters - an integrated approach from coreflood experiments through to foam diversion calculations. In: Presented at the IOR 2015 - 18th European Symposium on Improved Oil Recovery.

Kristiansen, T.S., Holt, T., 1992. Properties of flowing foam in porous media containing oil. In: Presented at the SPE/DOE Enhanced Oil Recovery Symposium. Society of Petroleum Engineers.

Nikolov, A.D., Wasan, D.T., Huang, D.W., Edwards, D.A., 1986. The effect of oil on foam stability: mechanisms and implications for oil displacement by foam in porous media. In: Presented at the SPE Annual Technical Conference and Exhibition. Society of Petroleum Engineers.

$\emptyset$ ren, P.-E., Bakke, S., 2003. Reconstruction of Berea sandstone and pore-scale modelling of wettability effects. J. Pet. Sci. Eng. Reserv. Wettability 39, 177-199. https://doi. org/10.1016/S0920-4105(03)00062-7.

Peksa, A.E., Wolf, K.-H.A.A., Zitha, P.L.J., 2015. Bentheimer sandstone revisited for experimental purposes. Mar. Pet. Geol. 67, 701-719. https://doi.org/10.1016/j. marpetgeo.2015.06.001.

Pugh, R.J., 1996. Foaming, foam films, antifoaming and defoaming. Adv. Colloid Interface Sci. 64, 67-142. https://doi.org/10.1016/0001-8686(95)00280-4.

Raza, S.H., 1970. Foam in porous media: characteristics and potential applications. Soc. Pet. Eng. J. 10, 328-336. https://doi.org/10.2118/2421-PA.

Schramm, L.L., Novosad, J.J., 1990. Micro-visualization of foam interactions with a crude oil. Colloids Surf. 46, 21-43. https://doi.org/10.1016/0166-6622(90)800467.

Schramm, L.L., Turta, A.T., Novosad, J.J., 1993. Microvisual and coreflood studies of foam interactions with a light crude oil. SPE Reserv. Eng. 8, 201-206. https://doi org/10.2118/20197-PA.

Simjoo, M., 2012. Immiscible Foam for Enhanced Oil Recovery (PhD Dissertation). Delft University of Technology.

Tang, 2019. The Effect of Oil on Foam for EOR (PhD Dissertation). Delft University of Technology.

Tang, J., Vincent-Bonnieu, S., Rossen, W., 2018. Experimental investigation of the effect of oil on steady-state foam flow in porous media. SPE J. https://doi.org/10.2118/ 194015-PA. 